\title{
Modified Lattice Landau Gauge
}

\section{Lorenz von Smekal;, Dhagash Mehta, André Sternbeck ${ }^{\dagger}$ and Anthony G. Williams}

Centre for the Subatomic Structure of Matter, School of Chemistry \& Physics

The University of Adelaide, Adelaide, SA 5005, Australia

E-mail: lorenz.smekaleadelaide.edu.au

We propose a modified lattice Landau gauge based on stereographically projecting the link variables on the circle $S^{1} \rightarrow \mathbb{R}$ for compact $U(1)$ or the 3 -sphere $S^{3} \rightarrow \mathbb{R}^{3}$ for $S U(2)$ before imposing the Landau gauge condition. This can reduce the number of Gribov copies exponentially and solves the Gribov problem in compact $U(1)$ where it is a lattice artifact. Applied to the maximal Abelian subgroup this might be just enough to avoid the perfect cancellation amongst the Gribov copies in a lattice BRST formulation for $S U(N)$, and thus to avoid the Neuberger 0/0 problem. The continuum limit of the Landau gauge remains unchanged.

The XXV International Symposium on Lattice Field Theory

July 30 - August 42007

Regensburg, Germany

\footnotetext{
${ }^{*}$ Speaker.

${ }^{\dagger}$ Supported by the Australian Research Council
} 


\section{Introduction}

The machinery for dealing with redundant degrees of freedom due to gauge invariance in local quantum field theory is based on BRST symmetry. In QED it reduces to the long-known GuptaBleuler construction. In non-Abelian gauge theories, all is well in perturbation theory also. Beyond that, however, there is a problem with such constructions that has not been fully and comprehensively addressed as yet. It relates to the famous Gribov ambiguity, the existence of Gribov copies that satisfy the Lorenz condition (or any other local gauge fixing condition) but are related by gauge transformations, and are thus physically equivalent. Lattice gauge theory provides a rigorous nonperturbative framework whose strength and beauty derives from the fact that gauge invariance is manifest and fixing a gauge is not required. However, in order to arrive at a non-perturbative definition of non-Abelian gauge theories in the continuum, from a lattice formulation, we need to be able to perform the continuum limit in a formally watertight way. When attempting to fix a gauge via BRST formulations on the lattice, the Gribov ambiguity manifests itself in the notorious Neuberger problem which asserts that the expectation value of any gauge invariant (and thus physical) observable in a lattice BRST formulation will always be of the indefinite form 0/0 [1].

One possible way forward is provided by the ghost/anti-ghost symmetric Curci-Ferrari gauges. While the Neuberger 0/0 problem can be extended to include these non-linear gauges with their extended double-BRST symmetry despite their quartic ghost self-interactions, they do allow the introduction of a mass term for ghosts. Such a Curci-Ferrari mass breaks the nilpotency of the BRST/anti-BRST charges which is known to result in a loss of unitarity and which perhaps therefore meant that this relatively old model received little attention for many years. On the other hand, this mass also serves to regulate the Neuberger zeroes in a lattice formulation. Expectation values of observables can then be meaningfully defined in the limit $m \rightarrow 0$ via l'Hospital's rule [2, 3, 4].

Here we propose an alternative more directly relating to the methods of topological quantum field theory on the gauge group [5,6]. The problem there is that the partition function of the topological model to be used as the gauge-fixing device computes a topological invariant such as the Euler characteristic of the gauge group which vanishes. In the Landau gauge, for example, this partition function reduces to the sign-weighted sum over all Gribov copies as the critical points of a Morse potential on the gauge orbit which corresponds to computing the Euler characteristic of the gauge group via the Poincaré-Hopf theorem. This is the limit of vanishing gauge parameter, $\xi \rightarrow 0$. Neuberger's $0 / 0$ argument is based on the opposite limit, $\xi \rightarrow \infty$. Even though there is no gauge-fixing in this limit in the first place, the topological model does not depend on $\xi$ and actually computes the same invariant. This can be seen most clearly in the ghost/anti-ghost symmetric Curci-Ferrari model for $S U(N)$, where in the massless case the gauge-fixing partition function for $\xi \rightarrow \infty$ factorises into a product of one Gauss-Bonnet integral expression for the vanishing Euler characteristic $\chi(S U(N))$ per each site of the lattice [4].

The $0 / 0$ problem due to the vanishing Euler characteristic of $S U(N)$ is avoided when fixing the gauge only up to the maximal Abelian subgroup $U(1)^{N-1}$ because the Euler characteristic of the coset manifold is non-zero. In Ref. [6] the corresponding lattice BRST has been explicitly constructed for $S U(2)$, where the coset manifold is the 2-sphere and $\chi(S U(2) / U(1))=\chi\left(S^{2}\right)=2$.

Is this an indication that the Neuberger problem might be solved when that of compact $U(1)$ is? The cancellation of Gribov copies is certainly present there already because $\chi\left(S^{1}\right)=0$. Here 
we propose a perhaps surprisingly simple solution to this problem by stereographically projecting the circle $S^{1} \rightarrow \mathbb{R}$ which can be achieved by a simple modification of the minimising potential. This then leads to a positive (semi)definite Faddeev-Popov operator, and there is thus no cancellation of Gribov copies in compact $U(1)$ with this so modified lattice Landau gauge.

The same procedure can be readily extended and applied to $S U(2)$ with the 3-sphere as group manifold and projecting $S^{3} \rightarrow \mathbb{R}^{3}$. Even though we don't have an explicit proof for $S U(2)$ (except in one dimension) that this solves the Neuberger problem, the remaining manifold relevant in the topological argument then becomes $\mathbb{R} P^{2}$ whose Euler characteristic is unity. For a lattice BRST construction based on this topological invariant we certainly do not expect that there are no contributions from any Gribov copies outside the first Gribov horizon (with both signs of the Faddeev-Popov determinant), as we know there are such copies in the continuum limit. The topological argument indicates, however, that once the cancellation in a one-parameter $U(1)$-subgroup direction along the gauge orbits is avoided, there will be no perfect cancellation anymore between remaining copies of different signs, as $\chi\left(\mathbb{R} P^{2}\right)=1$. Whether that is really sufficient to avoid the Neuberger problem or not, it is important to note that our modifications do not affect the continuum limit. One implementation of lattice Landau gauge is as well justified as the other as far as the continuum gauge theory is concerned. Any observable differences between the two are lattice artifacts. Luckily, as we will see by comparison, these differences appear to be surprisingly small.

\section{Modified lattice Landau gauge for compact $U(1)$}

To be specific, for compact $U(1)$ with link angles $\varphi_{i, \mu} \in(-\pi, \pi]$ and gauge transformations $\varphi_{i, \mu} \rightarrow \varphi_{i, \mu}^{\theta}=\varphi_{i, \mu}+\theta_{i+\mu}-\theta_{i} \bmod 2 \pi$ by gauge angles $\theta_{i} \in(-\pi, \pi]$ per site $i$ of the lattice, consider in parallel the $2 \pi$-periodic potentials on gauge orbits $\varphi$ defined by

$$
V_{\varphi}[\theta]=\sum_{i, \mu}\left(1-\cos \varphi_{i, \mu}^{\theta}\right), \text { and } \widetilde{V}_{\varphi}[\theta]=-2 \sum_{i, \mu} \ln \left(\left(1+\cos \varphi_{i, \mu}^{\theta}\right) / 2\right) .
$$

The first one is that of standard lattice Landau gauge corresponding to the height function on the circle per link as a proper Morse potential for the $U(1)$ gauge group on the lattice. Its critical points are the Gribov copies of standard lattice Landau gauge. In the modified potential $\widetilde{V}_{\varphi}$ we have introduced a singularity to suppress the South pole. Variation w.r.t. the gauge angles $\theta_{i}$ gives,

$$
f_{i}(\theta)=-\sum_{\mu}\left(\sin \varphi_{i, \mu}^{\theta}-\sin \varphi_{i-\mu, \mu}^{\theta}\right), \text { and } \tilde{f}_{i}(\theta)=-2 \sum_{\mu}\left(\tan \left(\varphi_{i, \mu}^{\theta} / 2\right)-\tan \left(\varphi_{i-\mu, \mu}^{\theta} / 2\right)\right) \text {. }
$$

The solutions to the Landau gauge conditions $f_{i}=0$ or $\tilde{f}_{i}=0$, for $i=1, \ldots$ (\#sites), define the set of Gribov copies in either case. To see the cancellation of Gribov copies in the standard case explicitly, consider the admittedly trivial example of compact $U(1)$ in 1 dimension as a simple warm-up exercise. Of course, there is no dynamics in 1 dimension as there is essentially only a single gauge orbit. Nevertheless, the number of solutions to the standard Landau gauge condition grows exponentially with the problem size. To see this, consider a finite chain with $n$ sites. To remove global gauge transformations, impose anti-periodic boundary conditions on the link and gauge angles (i.e., $C$-periodic b.c.'s for the corresponding group elements). With periodic b.c.'s the Faddeev-Popov matrix will, of course, always have one exact zero eigenvalue due to the unfixed global gauge invariance. This one remaining zero is otherwise inessential for the present argument. 
With a.p.b.c.'s the solutions to the conditions $f_{i}=0, i=1, \ldots n$, are then found to be simply given by sequences of link angles where every link $\varphi_{i}^{\theta}$ can only either be 0 or $\pi$, no matter what the initial set of link angles $\varphi_{i}$ is. There are $2^{n}$ such configurations which are Gribov copies of the trivial configuration $\varphi=0$ (i.e., $\varphi_{i}=0, i=1, \ldots n$ ). This is because the number of links equals the number of sites, in 1 dimension. With a.p.b.c.'s, every link configuration is gauge-equivalent to $\varphi=0$ (and gauge-equivalent up to a constant with p.b.c.'s). In the corresponding gauge-fixing partition function of standard lattice BRST,

$$
Z_{\mathrm{GF}}=\int_{V^{n}} \frac{d^{n} \theta}{(2 \pi)^{n}} \int_{\mathbb{R}^{n}} d^{n} b \operatorname{det} M_{\mathrm{FP}} \exp \left\{i(b, f)-\frac{\xi}{2}(b, b)\right\} \stackrel{\xi=0}{=} \int_{V^{n}} d^{n} \theta \operatorname{det} M_{\mathrm{FP}} \delta^{n}(f(\theta)),
$$

where $V^{n}$ is the integration range of the gauge angles $-\pi \leq \theta_{i} \leq \pi$ and $M_{\mathrm{FP}}$ is the Faddeev-Popov matrix, we can therefore perform a variable transform $\theta \mapsto s=\varphi+M \theta$, where $(M \theta)_{i}=\theta_{i+1}-\theta_{i}$. With a.p.b.c.'s, $|\operatorname{det} M|=2$ reflecting the fact that the map is 2 to 1 when applying modulo $2 \pi$ on $s$ (changing every gauge angle by $\pi$ leaves the link configuration unchanged $\bmod 2 \pi$ ). With periodic b.c.'s the matrix $M$ has one eigenvalue zero as we can't change the average value of the link angles by a gauge transformation. With this variable transform, we see that in the anti-periodic case the $2^{n}$ solutions $s^{(k)}$ (with components $s_{i}^{(k)}=0$ or $\pi$ ) are obtained from $\varphi=0$ with $s^{(k)}=M \theta^{(k)}$. Inverting this relation, we find two classes of solutions for $\theta \in V^{n}$ : (a) sequences with all $\theta_{i}$ in $\{0, \pi\}$ and (b) sequences with all $\theta_{i}$ in $\{\pi / 2,-\pi / 2\}$. There are thus together $2^{n+1}$ solutions to $f=0$ for the gauge angles $\theta$ in $V^{n}$ (the double counting of link configurations with $2 \pi$-periodicity corresponds to identifying pairs of $\theta$, related by a swapping of all components, in each of the 2 types separately). Type (a) is easily seen to always lead to a link configuration $s^{(k)}$ with an even number of $\pi$ 's, while type (b) always leads to on odd number of links in the South pole. We have,

$$
Z_{\mathrm{GF}}=2 \int_{V^{n}} d^{n} s|\operatorname{det} M|^{-1} \operatorname{det} M_{\mathrm{FP}} \delta^{n}(f(\theta(s)))=2 \sum_{k=1}^{2^{n}} \operatorname{sign}\left(\operatorname{det} M_{\mathrm{FP}}\left(s^{(k)}\right)\right) .
$$

The Faddeev-Popov matrix is obtained from

$$
\left(M_{\mathrm{FP}}\right)_{i j}=\frac{\partial f_{i}(\theta)}{\partial \theta_{j}}=-\cos s_{i} \delta_{i+1, j}+\left(\cos s_{i}+\cos s_{i-1}\right) \delta_{i, j}-\cos s_{i-1} \delta_{i-1, j},
$$

where we have used the variables $s_{i}=\varphi_{i}+\theta_{i+1}-\theta_{i}$ as above. It can be written in the form

$$
M_{\mathrm{FP}}=M^{T} D\left(\cos s_{1}, \cos s_{2}, \ldots \cos s_{n}\right) M,
$$

where $D\left(c_{1}, c_{2}, \ldots c_{n}\right)$ is a diagonal $n \times n$ matrix with entry $c_{i}$ at position $i$ along the diagonal. The real matrix $M$ for a.p.b.c.'s is the same non-singular matrix as above. From Sylverster's law of inertia, the numbers of positive, negative and zero eigenvalues of the Hermitian Faddeev-Popov matrix $M_{\mathrm{FP}}$ are the same as those of the diagonal matrix $D$. For the Gribov copies $s^{(k)}$ of our $U(1)$ chain with all $s_{i}^{(k)}=0$ or $\pi$ there are no zero eigenvalues. Moreover, we see that we have exactly one negative eigenvalue for every $\pi$ link in the chain. The number of $\pi$ 's is even and the FaddeevPopov determinant thus positive for all type (a) solutions, and negative for those of type (b) with an odd number of $\pi$ links. The number of copies of type (a) and (b) are the same, each with the $2^{n}$ gauge configurations $\theta$ in $V^{n}$ yielding $2^{n-1}$ inequivalent link configurations $\bmod 2 \pi$. Therefore,

$$
Z_{\mathrm{GF}}=2 \sum_{k=1}^{2^{n}} \operatorname{sign}\left(\operatorname{det} M_{\mathrm{FP}}\left(s^{(k)}\right)\right)=2\left(2^{n-1}-2^{n-1}\right)=0 .
$$


This is the Neuberger zero. Observe the $Z_{2}$ structure: we can pick any one solution of type (b) to define a gauge transformation which when applied to any copy of type (a) or (b) leads to a copy of the other type, and returns the original configuration when applied twice. For odd $n$ for example, the checkerboard gauge transformation $\theta=\left(\frac{\pi}{2},-\frac{\pi}{2}, \ldots \frac{\pi}{2}\right)$ changes every link by $\pi$ and therefore swaps the overall sign of $M_{\mathrm{FP}}$. Because $n$ is odd, this then also swaps the sign of the Faddeev-Popov determinant (the same works with periodic b.c.'s and even $n$ for $\theta=(0, \pi, 0, \ldots \pi)$; because there is one zero eigenvalue in the periodic case, the number of non-zero ones for even $n$ is odd and their product then changes sign also). It is very easy to see that this twofold symmetry amongst Gribov copies of opposite sign in fact exists in the standard Landau gauge for compact $U(1)$ in any dimension, and with it the Neuberger $0 / 0$ problem.

For our anti-periodic 1-dimensional model, the modified Landau gauge condition in Eq. (2.2), stereographically projecting the links $s_{i} \rightarrow \tan \left(s_{i} / 2\right)$, now analogously leads to a Faddeev-Popov matrix of the form

$$
\widetilde{M}_{\mathrm{FP}}=M^{T} D\left(\sec ^{2} \frac{s_{1}}{2}, \sec ^{2} \frac{s_{2}}{2}, \ldots \sec ^{2} \frac{s_{n}}{2}\right) M .
$$

Again, with a.p.b.c.'s, the real matrix $M$ is non-singular, while with periodic b.c.'s it has one zero eigenvalue. The diagonal elements of $D$ are all positive (in fact $\geq 1$ ) and so is $M_{\mathrm{FP}}$. Moreover, the Faddeev-Popov matrix in higher dimensions consists of a sum of terms of the same form, one for every dimension. As a sum of positive (semi-)definite matrices it remains such in any dimension.

The twofold symmetry among the solution that changes the sign of the Faddeev-Popov determinant no-longer exists. In 1 dimension, of the originally $2^{n+1}$ solutions only 2 remain. There are no type (b) solutions to $\tilde{f}=0$, and of type (a) only $\theta=(0,0, \ldots 0)$ and $(\pi, \pi, \ldots \pi)$ survive which both lead to the same trivial link configuration $\bmod 2 \pi$. There are thus no more Gribov copies. This changes in higher dimensions, where there are copies which, e.g., in 2 dimensions differ from each other by a number of vortex pairs. Nevertheless, the Faddeev-Popov matrix is (semi-)positive for any choice of $\theta$, not only for the solutions to $\tilde{f}=0$. This means that the potential $\widetilde{V}_{\varphi}[\theta]$ is convex to the above. It does have singularities, but there is always a unique minimum lying in between the infinite walls. The gauge-fixing partition function counts their number, i.e., the Gribov copies in this modified Landau gauge, which should not depend on the gauge orbit. This may sound strange at first, but it is quite possible in compact $U(1)$ where the copies (all minima here) can be classified entirely in terms of their vortex content [7]. It will not be the case for $S U(N)$ generalisations. ${ }^{1}$

Note that the singularities in $\widetilde{V}_{\varphi}[\theta]$ are crucial in order to escape Neuberger's argument. Consider the partition function of our modified model with an additional parameter $t$ as in [1],

$$
\begin{aligned}
\widetilde{Z}_{\mathrm{GF}}(t) & =\int_{V^{n}} \frac{d^{n} \theta}{(2 \pi)^{n}} \int_{\mathbb{R}^{n}} d^{n} b \operatorname{det}\left(t \widetilde{M}_{\mathrm{FP}}\right) \exp \left\{i t(b, \tilde{f})-\frac{\xi}{2}(b, b)\right\} \\
& =t^{n} \int_{V^{n}} \frac{d^{n} \theta}{(2 \pi \xi)^{n / 2}} \operatorname{det} \widetilde{M}_{\mathrm{FP}} \exp \left\{-\frac{t^{2}}{2 \xi}(\tilde{f}, \tilde{f})\right\}=t^{n} \sum_{\text {copies }} \int_{\mathbb{R}^{n}} \frac{d^{n} \tilde{f}}{(2 \pi \xi)^{n / 2}} \exp \left\{-\frac{t^{2}}{2 \xi}(\tilde{f}, \tilde{f})\right\} .
\end{aligned}
$$

Because $\operatorname{det} \widetilde{M}_{\mathrm{FP}}$ is always positive, it compensates the Jacobian of our variable transform $\theta \rightarrow \tilde{f}$. If $\tilde{f}$ was bounded, we could let $t \rightarrow 0$ and, from the prefactor $t^{n}$, observe the Neuberger zero. We can't do that anymore without encountering an infinity $\propto t^{-n}$ from the Gaussian integration over $\tilde{f}$

\footnotetext{
${ }^{1}$ Neither does it have to be because of the partial cancellations that then still occur, e.g., in computing $\chi\left(\mathbb{R} P^{2}\right)$.
} 

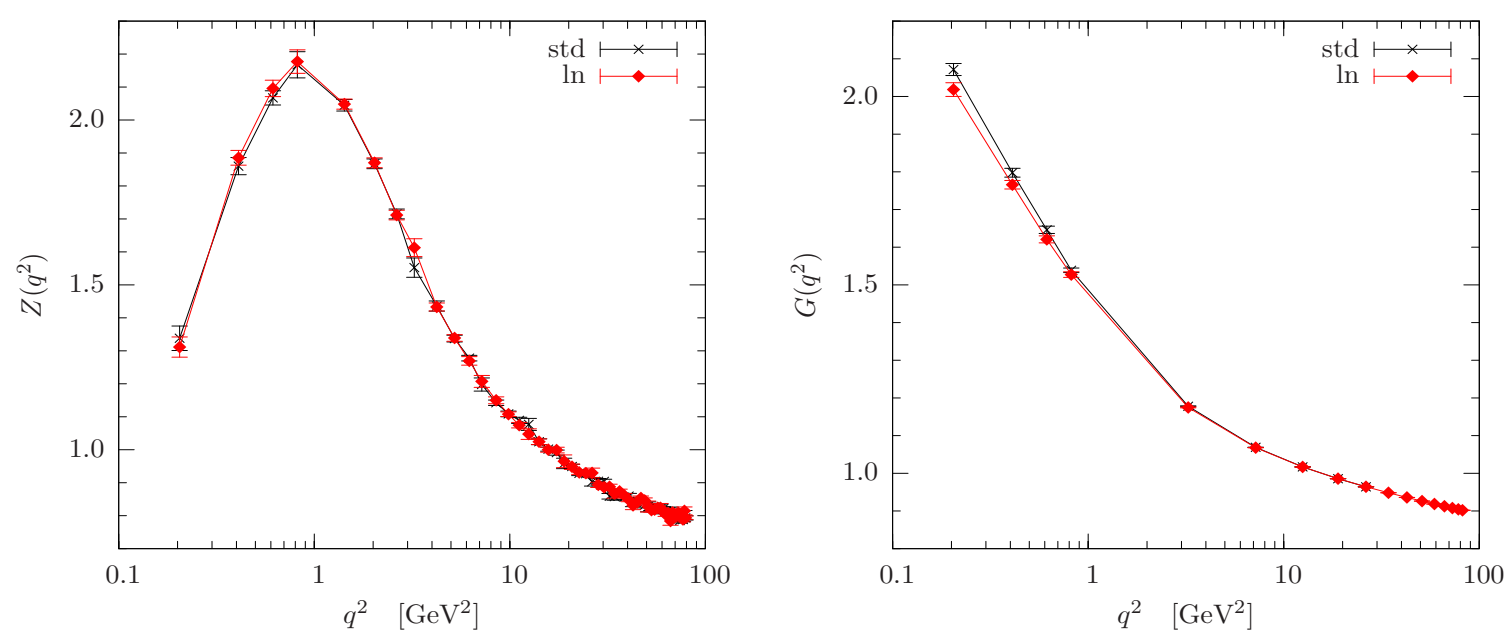

Figure 1: The gluon (left) and ghost (right) dressing functions for pure $\mathrm{SU}(2)$ gauge theory at $\beta=2.5$ on a $32^{4}$ lattice (renormalised at $\mu=4 \mathrm{GeV}$ ) with standard (std) and modified (ln) lattice Landau gauge-fixing.

because of the stereographic projection of the link angles in $\tilde{f}$ which decompactifies the integration range. The result is of course still $t$-independent and simply given by the number of Gribov copies,

$$
\widetilde{Z}_{\mathrm{GF}}(t)=(\# \text { copies })[=2 \text {, in } 1 \mathrm{dim} .], \text { independent of } t .
$$

This thus solves the Neuberger problem of compact $U(1)$ in any dimension.

\section{Stereographically projected lattice Landau gauge for $S U(2)$}

In order to analogously suppress the South pole, in the minimising potential for $S U(2)$, we essentially have to replace $\frac{1}{2} \operatorname{tr} U_{i, \mu}^{g} \rightarrow \ln \left(1+\frac{1}{2} \operatorname{tr} U_{i, \mu}^{g}\right)$ where $U_{i, \mu}^{g}=g_{i}^{\dagger} U_{i, \mu} g_{i+\mu}$ is the gauge transformed $\mu$-link at $i$, with $U, g \in S U(2)$. As above we thus compare the gauge-fixing potentials,

$$
V_{U}[g]=\frac{2}{\rho} \sum_{i, \mu}\left(1-\frac{1}{2} \operatorname{tr} U_{i, \mu}^{g}\right), \text { and } \quad \widetilde{V}_{U}[g]=-\frac{4}{\rho} \sum_{i, \mu} \ln \left(\frac{1}{2}+\frac{1}{4} \operatorname{tr} U_{i, \mu}^{g}\right) .
$$

With $U^{g}=x^{0}+i \sigma^{a} x^{a},\left(x^{0}, \vec{x}\right) \in S^{3}$, and stereographically projected $\tilde{x}^{a}=x^{a} /\left(1+x^{0}\right)$ as the corresponding variables in $\mathbb{R}^{3}$, the respective Landau gauge conditions become,

$$
f_{i}^{a}=-2 \sum_{\mu}\left(x_{i, \mu}^{a}-x_{i-\mu, \mu}^{a}\right), \text { and } \quad \tilde{f}_{i}^{a}=-4 \sum_{\mu}\left(\tilde{x}_{i, \mu}^{a}-\tilde{x}_{i-\mu, \mu}^{a}\right) .
$$

The modified Faddeev-Popov operator can then be obtained in the form, with $\tilde{x}^{0}=x^{0} /\left(1+x^{0}\right)$,

$$
\begin{aligned}
\left(\widetilde{M}_{\mathrm{FP}}\right)_{i j}^{a b}=2 \sum_{\mu}\{- & \left(\tilde{x}_{i, \mu}^{0} \delta^{a b}+\varepsilon^{a b c} \tilde{x}_{i, \mu}^{c}+\tilde{x}_{i, \mu}^{a} \tilde{x}_{i, \mu}^{b}\right) \delta_{i+\mu, j}+\left(\left(\tilde{x}_{i, \mu}^{0}+\tilde{x}_{i-\mu, \mu}^{0}\right) \delta^{a b}+\tilde{x}_{i, \mu}^{a} \tilde{x}_{i, \mu}^{b}\right. \\
& \left.\left.+\tilde{x}_{i-\mu, \mu}^{a} \tilde{x}_{i-\mu, \mu}^{b}\right) \delta_{i j}-\left(\tilde{x}_{i-\mu, \mu}^{0} \delta^{a b}-\varepsilon^{a b c} \tilde{x}_{i-\mu, \mu}^{c}+\tilde{x}_{i-\mu, \mu}^{a} \tilde{x}_{i-\mu, \mu}^{b}\right) \delta_{i-\mu, j}\right\} .
\end{aligned}
$$

The new feature, due to the logarithm in (3.1), are the $\tilde{x}^{a} \tilde{x}^{b}$-terms quadratic in the projected variables $\tilde{x}$. Apart from those, $\widetilde{M}_{F P}$ is of the same form as the standard Faddeev-Popov operator in $S U(2)$ with the $x$ 's replaced by $2 \tilde{x}$. The stereographically projected variables have the property that $\tilde{x}^{0}+\tilde{x} \cdot \tilde{x}=\frac{1}{2} \sec ^{2} \frac{\theta}{4}$, where $\frac{1}{2} \operatorname{tr} U^{g}=x_{0}=\cos \frac{\theta}{2}$ defines the azimuthal angle of the link in $S U(2)$. 
This can be used to show that $\widetilde{M}_{\mathrm{FP}}$ in (3.3) is still positive semi-definite when all links $\tilde{x}_{i}^{a}$ are aligned in the same $S U$ (2) direction. While this is true for all solutions to $\tilde{f}_{i}^{a}=0$ in (3.2) in 1 dimension, it is not generally the case, and it does therefore not follow that in higher dimensions $\widetilde{M}_{\mathrm{FP}}$ is generally a sum of positive terms, as it did in compact $U(1)$. The principal advantage of the stereographic projection is that we do not expect a perfect cancellation amongst Gribov copies of opposite sign as mentioned in the introduction. This would then allow to define a BRST formulation on the lattice.

The main point here of course is that we can eventually include all copies, not only minima, in a BRST-like average when using stereographic projection, which would produce a zero result in the standard case. This would then be analogous to the gauge-fixing procedure used in most functional continuum methods. This is not what we have done here. To assess the effect of the modification, as a first step, we calculated the dressing functions in the gluon and ghost propagators obtained from minimising each of the two potentials in (3.1). The results are compared in Fig. 1.

For the modified Landau gauge we used a specially adapted version of the Fourier-accelerated gauge fixing of [8]. The standard results and other details are those of [9] where we used overrelaxation. We calculated the gluon and ghost propagators on the same 50 starting configurations. After minimisation, however, the gauge-fixed configurations of one potential still show considerable violations of the other potential's condition. They are typically not nearly acceptable as solutions to the other gauge condition. Nevertheless, there are hardly any differences between the propagators obtained in each case. Even the differences observed in the ghost propagator at low momenta are so small that they might well be due to the different algorithms. The smallness of the effect might in fact seem surprising given that we invert the Hessian of different functions on different minima along the gauge orbits, but it is necessary for the equivalence of the two in the continuum limit.

\section{Conclusions}

Stereographic projection to decompactify link variables before gauge fixing solves the Neuberger $0 / 0$ problem in compact $U(1)$. We have good reason to expect it to prevent the cancellation of Gribov copies similarly in $S U(2)$. Generalisation to $S U(N)$ may not be entirely trivial but should also be possible. It is important to stress that the modifications do not affect the gauge group. The proposal is to set up a topological model that computes an invariant different from the vanishing Euler characteristic of the gauge group. This can be achieved by mapping the South pole to infinity to suppresses parts of a particular gauge orbit which should be of no harm but which is what fixing a gauge is all about. The continuum limit of the modified lattice Landau gauge remains unchanged.

ACK: Discussions with M. Schaden, Ph. de Forcrand and D. Zwanziger were greatly appreciated.

\section{References}

[1] H. Neuberger, Phys. Lett. B 175 (1986) 69; ibid. 183 (1987) 337.

[2] A. C. Kalloniatis, L. von Smekal and A. G. Williams, Phys. Lett. B 609 (2005) 424 [hep-lat/0501016].

[3] M. Ghiotti, L. von Smekal and A. G. Williams, AIP Conf. Proc. 892 (2007) 180 [hep-th/0611058].

[4] L. von Smekal, M. Ghiotti and A. G. Williams, "Decontracted double BRST on the lattice," in prep.

[5] L. Baulieu and M. Schaden, Int. J. Mod. Phys. A13 (1998) 985 [hep-th/9601039].

[6] M. Schaden, Phys. Rev. D 59 (1998) 014508 [hep-lat/9805020].

[7] See, e.g., N. Akino and J. M. Kosterlitz, Phys. Rev. B 66 (2002) 054536, and the references therein.

[8] C. T. H. Davies et al., Phys. Rev. D 37 (1988) 1581.

[9] A. Sternbeck, L. von Smekal, D. B. Leinweber, and A. G. Williams, PoS (LATTICE 2007) 340. 